\title{
How Childhood Maltreatment Is Related to Suicidality, Bipolarity and Central Serotonergic Activity in Patients with Major Depressive Disorder: A Cross-Sectional Pilot Study
}

\author{
Bun-Hee Lee ${ }^{1}$ and Young-Min Park ${ }^{2} \bowtie$ \\ ${ }^{1}$ Department of Psychiatry, Seoul Eunpyeong Hospital, Seoul, Republic of Korea \\ ${ }^{2}$ Department of Psychiatry, Inje University College of Medicine, Goyang, Republic of Korea
}

\begin{abstract}
Objective The aims of this study were to determine whether childhood maltreatment contributes to the occurrence of major depressive disorder (MDD) with bipolarity, and whether there is a relationship between central serotonergic activity, as assessed using loudness dependence of auditory evoked potentials (LDAEP), and childhood maltreatment.

Methods Thirty-five MDD patients were stratified according to the presence or absence of childhood trauma into two subgroups, childhood trauma (CT) and no childhood trauma (NCT), using the Korean version of the Childhood Trauma Questionnaire (K-CTQ). The CT group was subjected to further analysis. Several psychometric ratings were also applied. In addition, auditory processing for the loudness dependence of auditory evoked potentials (LDAEP), which was used as a marker of serotonergic activity, was measured before beginning medication.

Results There was a significant difference in total Korean Bipolar Spectrum Disorder Scale score between the CT and NCT groups $(\mathrm{t}=-2.14, \mathrm{p}=0.04)$. The total K-CTQ score was positively correlated with the total Beck Scale for Suicidal Ideation (BSS) score ( $\mathrm{r}=0.36$, $\mathrm{p}=0.036)$. In particular, emotional abuse was positively correlated with the total Barratt Impulsiveness Scale $(\mathrm{r}=0.38, \mathrm{p}=0.026), \mathrm{BSS}$ $(\mathrm{r}=0.38, \mathrm{p}=0.025)$, and Hamilton Depression Rating Scale (HAMD) $(\mathrm{r}=0.36, \mathrm{p}=0.035)$ scores. There was also a positive correlation between LDAEP and total Hypomania Personality Scale $(r=0.49, \mathrm{p}=0.02)$ and HAMD $(r=0.58, \mathrm{p}=0.004)$ scores within CT group.

Conclusion The findings of this study support that there is a relationship between childhood maltreatment and bipolarity in patients with MDD.

Psychiatry Investig 2016;13(2):190-195
\end{abstract}

Key Words Bipolarity, Childhood maltreatment, Trauma, LDAEP, Major depressive disorder, Bipolar disorder.

\section{INTRODUCTION}

Childhood maltreatment, including sexual abuse (SA), physical abuse (PA), emotional abuse (EA), physical neglect $(\mathrm{PN})$, and emotional neglect $(\mathrm{EN})$, is one of the most severe public-health problems worldwide. ${ }^{1}$ Childhood maltreatment is known to affect mental health in later adulthood; ${ }^{2}$ it is related to increased risk of major depressive disorder (MDD), bipolar disorder (BD), anxiety disorder, and alcohol abuse in adult-

Received: June 10, 2015 Revised: July 22, 2015

Accepted: August 8, 2015 Available online: January 11, 2016

$\triangle$ Correspondence: Young-Min Park, MD, PhD

Department of Neuropsychiatry, Inje University College of Medicine, 170 Juhwaro, Ilsanseo-gu, Goyang 10380, Republic of Korea

Tel: +82-31-910-7260, Fax: +82-31-910-7268, E-mail: medipark@hanmail.net

(a) This is an Open Access article distributed under the terms of the Creative Commons Attribution Non-Commercial License (http://creativecommons.org/licenses/by$\mathrm{nc} / 3.0$ ) which permits unrestricted non-commercial use, distribution, and reproduction in any medium, provided the original work is properly cited. hood. ${ }^{3,4}$ In the case of MDD, significant interactions with additive and recessive 5-HTTLPR (which encodes the serotonin transporter) genetic models were found for overall severity of childhood maltreatment. ${ }^{5} \mathrm{Li}$ and colleagues found that in addition to MDD, a significant number of patients with BD reported experience of childhood abuse and neglect. ${ }^{6} \mathrm{~A}$ recent meta-analysis also revealed that physically abused, emotionally abused, and neglected individuals have a higher risk of developing depressive disorders than their nonabused counterparts. ${ }^{2}$ This childhood maltreatment seems to reduce central serotonergic neurotransmission and influence the onset of psychiatric disorder in later adulthood..$^{-9}$

The loudness dependence of auditory evoked potentials (LDAEP), which is measured using auditory processing, has recently been introduced as a metric of serotonin activity. ${ }^{10}$ The results of preclinical/animal research have indicated the LDAEP is a reliable indicator of central serotonergic activity 
in mood disorder. ${ }^{11}$ The LDAEP has been identified as being inversely associated with central serotonergic activity, with a large LDAEP reflecting low serotonergic neurotransmission and vice versa. ${ }^{12}$ Thus, it was hypothesized that depressed patients with childhood maltreatment affected central serotonin neurotransmission, as assessed using LDAEP. In addition, to date there have been no reports of a relationship between childhood maltreatment and MDD with bipolarity or bipolar spectrum disorder, which has no manic or hypomanic episode but has the characteristics of BD. ${ }^{13}$ Patients exhibiting MDD with bipolarity have more psychopathology features, including impulsive ty and suicidal ideation, than those with MDD without bipolarity. ${ }^{14,15}$

The aims of the present study were to determine whether childhood maltreatment contributes to the occurrence of MDD with bipolarity, and whether there is a relationship between central serotonergic activity, as reflected by LDAEP, and childhood maltreatment. This is the first study to evaluate the relationship between childhood maltreatment and bipolarity.

\section{METHODS}

In total, 35 outpatients aged between 18 and 65 years who met the Diagnostic and Statistical Manual of Mental Disorders (DSM-IV)-text revision criteria for MDD were screened at Ilsan Paik Hospital between 2013 and 2014. Subjects who had psychotic symptoms, any additional mental disorders on axis I or II of the DSM-IV were excluded in order to remove bias except alcohol abuse. None of the subjects had a history of hypomanic or manic episodes. The following inclusion criteria were applied to enroll MDD patients in this study: 1) no history of antidepressant treatment within 3 months before first visiting our hospital, and 2) having total Beck Depression Inventory $(\mathrm{BDI})^{16}$ and Hamilton Depression Rating Scale (HAMD) ${ }^{17}$ scores exceeding 19 and 16, respectively, prior to the treatment (moderate-severe depression). Auditory processing for the baseline LDAEP, which was used as a marker of serotonergic activity in our previous study, ${ }^{14}$ was evaluated by measuring the auditory event-related potential before beginning medication with serotonergic agents.

The cohort was stratified into two subgroups according to the presence of childhood trauma (CT group) and no childhood trauma (NCT group), using the Korean version of the Childhood Trauma Questionnaire (K-CTQ), ${ }^{18,19}$ which classified patients as positive for a history of childhood maltreatment based on mild to moderate cutoff scores of the total KCTQ score (i.e., 41 points). ${ }^{20}$ The K-CTQ is a 28 -item selfreport questionnaire that consists of five subscales of childhood maltreatment experience: PA, EA, SA, PA, and EN. Each subscale comprises five items that are rated on a 5-point Likert scale, from 1 (never true) to 5 (very often true). In addition, It is appropriate for adults as well as adolescents (aged 12 and over). The entire cohort was also stratified into the following two subgroups according to whether they had suffered no maltreatment or had suffered one or more types of maltreatment (i.e., no vs one or more maltreatments) based on the moderateto-severe cutoff scores of five subscales: EA, $\geq 13$; PA, $\geq 10$; SA, $\geq 8$; $\mathrm{EN}, \geq 15$; and $\mathrm{PN}, \geq 10$. $^{20}$

Depression severity was assessed using HAMD and BDI. The Barratt Impulsiveness Scale (BIS), ${ }^{21}$ Hamilton Anxiety Scale (HAMA), ${ }^{22}$ Beck Scale for Suicidal Ideation (BSS), ${ }^{23}$ Beck Hopelessness Scale (BHS), ${ }^{24}$ Hypomania Personality Scale (HPS), ${ }^{25}$ and the Korean version of the Bipolar Spectrum Disorder Scale (K-BSDS $)^{26,27}$ were also applied. In this study, the K-BSDS was used to classify patients as positive for bipolarity based on moderately probable cutoff scores (i.e., 13 points). ${ }^{26}$ The CT group was subjected to further analysis. The study protocol was approved by the ethics committee of Inje University Ilsan Paik Hospital, and written informed consent to participate was obtained from all patients before beginning the investigation. ${ }^{25}$

\section{RESULTS}

A comparison of the demographic and clinical variables between the CT and NCT groups, defined according to the total $\mathrm{K}-\mathrm{CTQ}$ score, is presented in Table 1. The mean total K-BSDS score differed significantly between the NCT group (11.25) and the CT group (15.45; $\mathrm{t}=-2.14, \mathrm{p}=0.04)$. With the exception of $\mathrm{SA}$, the mean scores of each subscale were significantly higher in the CT than in the NCT group. The number of subjects with alcohol abuse was significantly higher in the CT than in the NCT group ( $\mathrm{p}=0.024$ ), as was the number of subjects with bipolarity $(\mathrm{p}=0.008)$.

Table 2 presents the demographic and clinical variables among the subjects stratified according to the number of maltreatments suffered, defined according to the cutoff scores for the five K-CTQ subscales (i.e., EN, EA, PN, PA, and SA). The mean total BSS, BHS, and K-BSDS scores were significantly higher in the groups with one or more childhood maltreatments than in that without childhood maltreatment (BSS: $\mathrm{t}=-2.10$, $\mathrm{p}=0.043$; BHS: $\mathrm{t}=-2.52, \mathrm{p}=0.017$; K-BSDS: $\mathrm{t}=-2.94, \mathrm{p}=0.0061$ ). The mean total K-CTQ scores and of each subscale, with the exception of SA, and the number of subjects with bipolarity ( $p=0.013$ ) were significantly higher in the group with one or more childhood maltreatment than in the group without childhood maltreatment.

The total K-CTQ score was positively correlated with the total BSS score $(\mathrm{r}=0.36, \mathrm{p}=0.036)$ (Figure 1$)$. EA was positively correlated with the total BIS $(r=0.38, p=0.026)$, BSS $(r=0.38$, 
$\mathrm{p}=0.025)$, and HAMD ( $\mathrm{r}=0.36, \mathrm{p}=0.035)$ scores. Patients with EA had higher BSS scores than those without EA $(t=-2.77$, $\mathrm{p}=0.009)$. EN was also positively correlated with the total BIS score $(r=0.35, p=0.042)$. Finally, the number of subjects with alcohol abuse was also significantly higher in the PA group than in the group without PA $(\mathrm{p}=0.013)$.

Further analysis of the CT group $(n=22)$ total K-CTQ score was performed. There was a positive correlation between LDAEP and HPS score $(\mathrm{r}=0.49, \mathrm{p}=0.02)$, but no correlation between LDAEP and K-BSDS score ( $\mathrm{r}=0.37, \mathrm{p}=0.091)$. In addition, there was a positive correlation between LDAEP and HAMD score ( $\mathrm{r}=0.58, \mathrm{p}=0.004$ ) (Figure 2). The total K-CTQ score was positively correlated with the total BDI score $(r=0.46$, $\mathrm{p}=0.031)$. Finally, EA was positively correlated with the total BDI $(r=0.48, p=0.021)$, BSS $(r=0.56, p=0.007)$, and HAMA $(\mathrm{r}=0.49, \mathrm{p}=0.022)$ scores.

\section{DISCUSSION}

This is the first pilot study to evaluate the relationship be- tween childhood maltreatment and bipolarity, not BD. The patients in the CT group had a higher K-BSDS score than did those in the NCT group, although they had no hypomanic or manic episodes in their life and were not satisfied with even BD NOS. This means that childhood maltreatment or trauma is associated with both increased risk of bipolarity and BD. In addition, Ghaemi and colleagues proposed a definition for those patients who fall in the middle of the mood spectrum, between the classic unipolar and type I bipolar extremes, although it was controversial. ${ }^{13,28}$ Thus, the present results may provide a clue as to the existence of a bipolar spectrum. Previous studies have already shown that childhood maltreatment was associated with an early age of onset, poor clinical course, suicidality, and substance abuse disorder in patients with BD. ${ }^{29}$ It was recently reported that exposure to childhood maltreatment potentiated the effects of recent stressors on adult mania. ${ }^{30}$ It was also reported that a significant number of patients with BD reported childhood maltreatment. ${ }^{6}$ Furthermore, compared to patients with unipolar depression, those with bipolar depression exhibited more suicidality, substance

Table 1. Comparison of demographic and clinical variables between the childhood trauma (CT) and no childhood trauma (NCT) groups, defined according to the cutoff ( 41 points) for the total Korean version of the Childhood Trauma Scale (K-CTQ) score

\begin{tabular}{|c|c|c|c|}
\hline Variable & $\operatorname{NCT}(\mathrm{N}=13)$ & $\mathrm{CT}(\mathrm{N}=22)$ & $\mathrm{p}$ \\
\hline Age (years) & $39.00 \pm 13.36$ & $36.68 \pm 12.65$ & 0.61 \\
\hline Sex (male/female) & $0 / 13$ & $5 / 17$ & 0.063 \\
\hline Suicide attempt hx (no/yes) ${ }^{\dagger}$ & $8 / 5$ & $13 / 9$ & 0.89 \\
\hline Alcohol abuse hx (no/yes) ${ }^{\dagger}$ & $8 / 5$ & $4 / 18$ & $<0.05^{*}$ \\
\hline Onset (years) & $32.08 \pm 12.95$ & $30.14 \pm 12.51$ & 0.66 \\
\hline $\operatorname{LDAEP}(\mu \mathrm{V} / \mathrm{dB})$ & $1.24 \pm 0.89$ & $0.94 \pm 0.73$ & 0.28 \\
\hline Total BIS score & $75.92 \pm 19.05$ & $82.55 \pm 12.77$ & 0.23 \\
\hline Total BDI score & $29.38 \pm 10.87$ & $30.0 \pm 8.79$ & 0.86 \\
\hline Total HAMD score & $16.54 \pm 5.90$ & $19.23 \pm 3.0$ & 0.12 \\
\hline Total BSS score & $10.23 \pm 11.20$ & $14.82 \pm 9.46$ & 0.20 \\
\hline Total BHS score & $10.15 \pm 8.20$ & $12.68 \pm 5.34$ & 0.33 \\
\hline Total HAMA score & $18.62 \pm 6.98$ & $20.82 \pm 4.36$ & 0.26 \\
\hline Total K-BSDS score & $11.25 \pm 5.38$ & $15.45 \pm 5.53$ & $<0.05^{*}$ \\
\hline Bipolarity (no/yes) ${ }^{\dagger}$ & $8 / 4$ & $4 / 18$ & $<0.01^{* *}$ \\
\hline Total HPS score & $52.75 \pm 6.96$ & $56.50 \pm 5.95$ & 0.11 \\
\hline Total CTQ score & $31.69 \pm 5.11$ & $58.36 \pm 12.73$ & $<0.01^{* *}$ \\
\hline EN score & $7.00 \pm 2.94$ & $17.05 \pm 5.68$ & $<0.01^{* *}$ \\
\hline PA score & $6.15 \pm 1.95$ & $10.36 \pm 4.52$ & $<0.01^{* *}$ \\
\hline SA score & $5.38 \pm 0.96$ & $6.64 \pm 3.54$ & 0.22 \\
\hline EA score & $5.85 \pm 1.34$ & $12.18 \pm 3.33$ & $<0.01^{* *}$ \\
\hline PN score & $7.31 \pm 2.10$ & $12.14 \pm 4.44$ & $<0.01^{* *}$ \\
\hline
\end{tabular}

Comparisons were made by independent t-test. ${ }^{*} \mathrm{p}<0.05,{ }^{* *} \mathrm{p}<0.01,{ }^{\dagger}$ chi-square test. BIS: Barratt Impulsiveness Scale, BHS: Beck Hopelessness Scale, BSS: Beck Scale for Suicidal Ideation, K-BSDS: Korean version of the Bipolar Spectrum Disorder Scale, EN: emotional neglect, EA: emotional abuse, HAMD: Hamilton Depression Rating Scale, HAMA: Hamilton Anxiety Scale, HPS: Hypomania Personality Scale, hx: history, LDAEP: loudness dependence of auditory evoked potentials, PA: physical abuse, PN: physical neglect, SA: sexual abuse 
Table 2. Comparison of demographic and clinical variables between the groups with and without any kind of childhood trauma defined according to cutoffs for five K-CTQ subscales

\begin{tabular}{|c|c|c|c|}
\hline Variable & No. of childhood trauma $(\mathrm{N}=9)$ & Childhood trauma $(\geq 1)(\mathrm{N}=26)$ & $\mathrm{p}$ \\
\hline Age (years) & $37.44 \pm 15.02$ & $37.84 \pm 12.42$ & 0.93 \\
\hline Sex (male/female) & $0 / 9$ & $5 / 21$ & 0.16 \\
\hline Suicide attempt hx (no/yes) ${ }^{\dagger}$ & $6 / 3$ & $15 / 11$ & 0.64 \\
\hline Alcohol abuse hx (no/yes) ${ }^{\dagger}$ & $5 / 4$ & $7 / 19$ & 0.12 \\
\hline Onset (years) & $32.44 \pm 15.80$ & $30.31 \pm 11.49$ & 0.67 \\
\hline $\operatorname{LDAEP}(\mu \mathrm{V} / \mathrm{dB})$ & $1.26 \pm 0.98$ & $0.99 \pm 0.73$ & 0.39 \\
\hline Total BIS score & $73.38 \pm 15.76$ & $82.31 \pm 14.88$ & 0.15 \\
\hline Total BDI score & $26.78 \pm 10.64$ & $30.81 \pm 9.02$ & 0.28 \\
\hline Total HAMD score & $16.00 \pm 6.06$ & $19.00 \pm 4.28$ & 0.11 \\
\hline Total BSS score & $7.22 \pm 10.05$ & $15.15 \pm 9.65$ & $<0.05^{*}$ \\
\hline Total BHS score & $7.33 \pm 7.53$ & $13.27 \pm 5.55$ & $<0.05^{*}$ \\
\hline Total HAMA score & $17.56 \pm 6.69$ & $20.85 \pm 4.8$ & 0.12 \\
\hline Total K-BSDS score & $9.25 \pm 4.23$ & $15.42 \pm 5.43$ & $<0.01^{* *}$ \\
\hline Bipolarity (no/yes) ${ }^{\dagger}$ & $6 / 2$ & $6 / 20$ & $<0.05^{*}$ \\
\hline Total HPS score & $51.75 \pm 8.40$ & $56.23 \pm 5.54$ & 0.087 \\
\hline Total K-CTQ score & $29.56 \pm 3.91$ & $55.00 \pm 14.25$ & $<0.01^{* *}$ \\
\hline EN score & $6.33 \pm 2.29$ & $15.73 \pm 6.24$ & $<0.01^{* *}$ \\
\hline PA score & $5.22 \pm 0.67$ & $10.04 \pm 4.29$ & $<0.01^{* *}$ \\
\hline SA score & $5.22 \pm 0.67$ & $6.50 \pm 3.30$ & 0.262 \\
\hline EA score & $5.78 \pm 1.09$ & $11.23 \pm 3.87$ & $<0.01^{* *}$ \\
\hline PN score & $7.00 \pm 1.94$ & $11.50 \pm 4.44$ & $<0.01^{* *}$ \\
\hline
\end{tabular}

Comparisons were made by independent t-test. ${ }^{*} \mathrm{p}<0.05,{ }^{* *} \mathrm{p}<0.01,{ }^{\dagger}$ chi-square test. BIS: Barratt Impulsiveness Scale, BHS: Beck Hopelessness Scale, BSS: Beck Scale for Suicidal Ideation, K-BSDS: Korean version of the Bipolar Spectrum Disorder Scale, EN: emotional neglect, EA: emotional abuse, HAMD: Hamilton Depression Rating Scale, HAMA: Hamilton Anxiety Scale, HPS: Hypomania Personality Scale, hx: history, LDAEP: loudness dependence of auditory evoked potentials, PA: physical abuse, PN: physical neglect, SA: sexual abuse

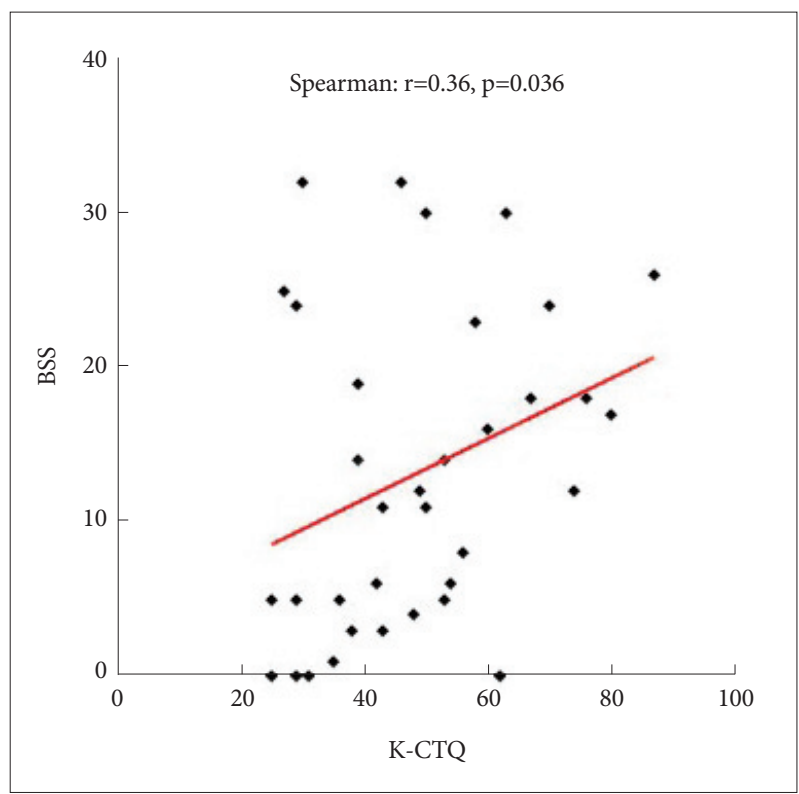

Figure 1. Correlation between the Korean version of the Childhood Trauma Questionnaire (K-CTQ) and Beck Scale for Suicidal Ideation (BSS).

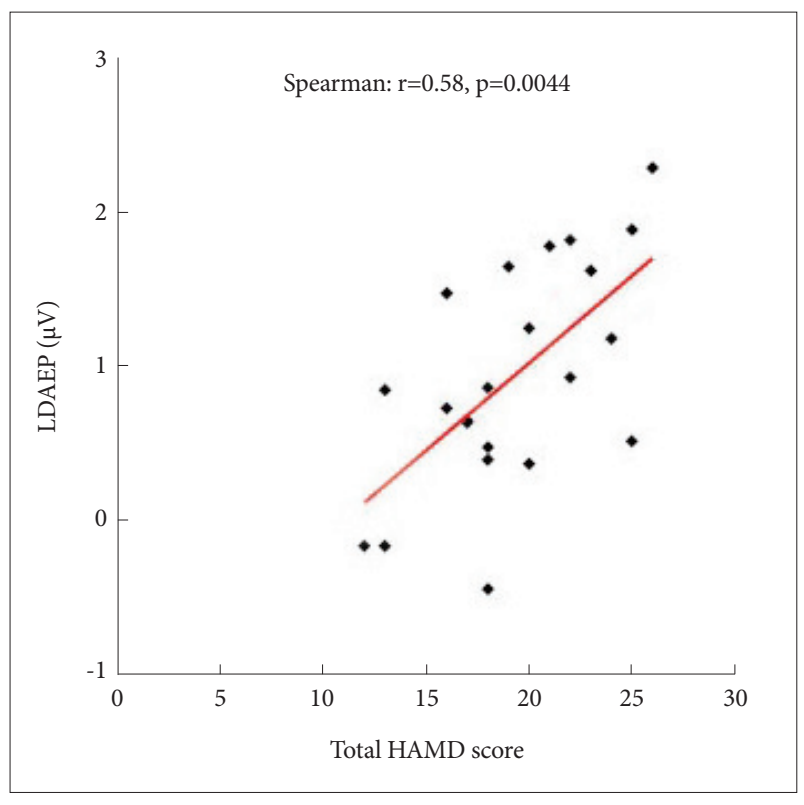

Figure 2. Correlation between the loudness dependence of auditory evoked potentials (LDAEP) and Hamilton Depression Rating Scale (HAMD) score within the group with childhood trauma. 
abuse, and impulsivity. ${ }^{28}$ In the present study, patients with one or more kinds of maltreatment had higher total BSS and BHS scores, and higher total K-BSDS scores as compared to MDD patients without any kind of maltreatment. This suggests that childhood maltreatment contributes to severe bipolarity or suicidality.

The total K-CTQ score was positively correlated with the total BSS score, and in particular, EA was positively correlated with the total BIS, BSS, and HAMD scores. These results show that the existence of childhood maltreatment was associated with worsening severity of illness and clinical course. Similarly, previous studies have demonstrated that childhood maltreatment is associated with the occurrence of psychiatric illness, including $\mathrm{BD}$ and $\mathrm{MDD}$, with a potentially dose-dependent effect. ${ }^{31}$ Another study revealed that childhood trauma, including abuse or neglect, was associated with an increased risk of incident and recurrent mania, and that childhood trauma was associated with age at onset, and symptoms of postraumatic stress disorder and anxiety. ${ }^{6}$

Further analysis of the CT group revealed a positive correlation between EA and total HAMA and BDI scores. Thus, childhood maltreatment appears to affect anxiety levels in some individuals, as shown in another recent study. ${ }^{6}$ Regarding central serotonergic activity within the CT group, as depression severity elevated, the LDAEP also increased; in other words, central serotonergic activity decreased as the severity of depression increased. Similarly, previous study have shown that the LDAEP in patients is related to the severity of their depression, such that the more depressive they are, the stronger is their LDAEP, which reflects lower central serotonergic activity. In another study it was also indicated that there is a negative correlation between serotonergic activity and depression severity. ${ }^{14}$ In addition, some studies have demonstrated the existence of a relationship between childhood maltreatment and the serotonin system. A recent study found that the short variant of the serotonin transporter gene (5-HTTLPR) specifically sensitizes the effect of childhood maltreatment on whether adultonset depression leads to a chronic course. ${ }^{8}$ An interaction between 5-HTTLPR and a specific early environmental risk, such as SA or PA, has also been demonstrated in recurrent depressive disorder. ${ }^{5}$ Regarding central serotonergic activity within the CT group, as the HPS score increased, central serotonergic activity decreased, indicating that stronger bipolarity reflects lower serotonergic activity. In line with the present findings, Young and colleagues found decreased serotonin metabolite levels and turnover in patients with BD. ${ }^{32}$ However, BSDS score was not correlated with central serotonergic activity unlike HPS score in the present study. In addition, Wiste and colleagues found both norepinephrine and serotonin activities were lower in six suicides with BD compared to those with unipolar major depression. ${ }^{33}$ However, other studies have produced controversial and contradictory results. ${ }^{34,35}$

The small sample in the present study limits the generalizability of the results. In addition, the K-BSDS is used only as a screening tool to help identify bipolarity, and not to analyze bipolarity itself. Notwithstanding these limitations, this is the first study to evaluate the relationship between childhood maltreatment and bipolarity.

In conclusion, the findings of this study support that there is a relationship between childhood maltreatment and bipolarity in patients with MDD. In other words, childhood trauma can be associated with both increased risk of bipolarity and BD. In future, more studies with larger cohorts are needed to confirm the findings.

\section{Acknowledgments}

This study was supported by a grant from National Research Foundation of Korea (NRF), funded by Ministry of Education (NRF-2014R1A1A2A 10059345). The authors would like to thank Jo BW for her assistance with data collection.

\section{REFERENCES}

1. Pereda N, Guilera G, Forns M, Gomez-Benito J. The prevalence of child sexual abuse in community and student samples: a meta-analysis. Clin Psychol Rev 2009;29:328-338.

2. Norman RE, Byambaa M, De R, Butchart A, Scott J, Vos T. The longterm health consequences of child physical abuse, emotional abuse, and neglect: a systematic review and meta-analysis. PLoS Med 2012;9: e1001349.

3. Arnow BA. Relationships between childhood maltreatment, adult health and psychiatric outcomes, and medical utilization. J Clin Psychiatry 2004;65(Suppl 12):10-15.

4. Sachs-Ericsson N, Cromer K, Hernandez A, Kendall-Tackett K. A review of childhood abuse, health, and pain-related problems: the role of psychiatric disorders and current life stress. J Trauma Dissociation 2009;10:170-188.

5. Fisher HL, Cohen-Woods S, Hosang GM, Korszun A, Owen M, Craddock N, et al. Interaction between specific forms of childhood maltreatment and the serotonin transporter gene (5-HTT) in recurrent depressive disorder. J Affect Disord 2013;145:136-141.

6. Li XB, Liu JT, Zhu XZ, Zhang L, Tang YL, Wang CY. Childhood trauma associates with clinical features of bipolar disorder in a sample of Chinese patients. J Affect Disord 2014;168C:58-63.

7. Berglund KJ, Balldin J, Berggren U, Gerdner A, Fahlke C. Childhood maltreatment affects the serotonergic system in male alcohol-dependent individuals. Alcohol Clin Exp Res 2013;37:757-762.

8. Miller JM, Kinnally EL, Ogden RT, Oquendo MA, Mann JJ, Parsey RV. Reported childhood abuse is associated with low serotonin transporter binding in vivo in major depressive disorder. Synapse 2009;63:565-573.

9. Roy A. Self-rated childhood emotional neglect and CSF monoamine indices in abstinent cocaine-abusing adults: possible implications for suicidal behavior. Psychiatry Res 2002;112:69-75.

10. Hegerl U, Juckel G. Intensity dependence of auditory evoked potentials as an indicator of central serotonergic neurotransmission: a new hypothesis. Biol Psychiatry 1993;33:173-187.

11. Hegerl U, Gallinat J, Juckel G. Event-related potentials. Do they reflect central serotonergic neurotransmission and do they predict clinical response to serotonin agonists? J Affect Disord 2001;62:93-100.

12. Juckel G, Hegerl U, Molnar M, Csepe V, Karmos G. Auditory evoked 
potentials reflect serotonergic neuronal activity--a study in behaving cats administered drugs acting on 5-HT1A autoreceptors in the dorsal raphe nucleus. Neuropsychopharmacology 1999;21:710-716.

13. Ghaemi SN. Bipolar spectrum: a review of the concept and a vision for the future. Psychiatry Investig 2013;10:218-224.

14. Park YM, Lee SH. Can the loudness dependence of auditory evoked potentials and suicidality be used to differentiate between depressive patients with and without bipolarity. Psychiatry Investig 2013;10:143-147.

15. Park YM, Lee SH. Clinical usefulness of Loudness Dependence of Auditory Evoked Potentials (LDAEP) in patients with bipolar disorder. Psychiatry Investig 2013;10:233-237.

16. Beck AT, Ward CH, Mendelson M, Mock J, Erbaugh J. An inventory for measuring depression. Arch Gen Psychiatry 1961;4:561-571.

17. Hamilton M. A rating scale for depression. J Neurol Neurosurg Psychiatry 1960;23:56-62.

18. Bernstein DP, Stein JA, Newcomb MD, Walker E, Pogge D, Ahluvalia T, et al. Development and validation of a brief screening version of the Childhood Trauma Questionnaire. Child Abuse Negl 2003;27:169-190.

19. Yu JH, Park JS, Park DH, Ryu SH, Ha JH. Validation of the Korean Childhood Trauma Questionnaire: the practical use in couselling and therapeutic intervention. Kor J Health Psychol 2009;14:563-578.

20. Bernstein D, Fink L. Childhood Trauma Questionnaire: A Retrospective Self-Report Questionnaire and Manual. San Antonio, TX: Psychological Corp; 1998.

21. Patton JH, Stanford MS, Barratt ES. Factor structure of the Barratt impulsiveness scale. J Clin Psychol 1995;51:768-774.

22. Maier W, Buller R, Philipp M, Heuser I. The Hamilton Anxiety Scale: reliability, validity and sensitivity to change in anxiety and depressive disorders. J Affect Disord 1988;14:61-68.

23. Beck AT, Kovacs M, Weissman A. Assessment of suicidal intention: the Scale for Suicide Ideation. J Consult Clin Psychol 1979;47:343-352.

24. Beck AT, Weissman A, Lester D, Trexler L. The measurement of pessimism: the hopelessness scale. J Consult Clin Psychol 1974;42:861-865.
25. Schalet BD, Durbin CE, Revelle W. Multidimensional structure of the Hypomanic Personality Scale. Psychol Assess 2011;23:504-522.

26. Nassir Ghaemi S, Miller CJ, Berv DA, Klugman J, Rosenquist KJ, Pies RW. Sensitivity and specificity of a new bipolar spectrum diagnostic scale. J Affect Disord 2005;84:273-277.

27. Wang HR, Kim SK, Kang SH, Joo YH, Kim CY. The validation study of the Korean version of the bipolar spectrum diagnostic scale. J Korean Neuropsychiatr Assoc 2008;47:533-539.

28. Ghaemi SN, Ko JY, Goodwin FK. "Cade's disease" and beyond: misdiagnosis, antidepressant use, and a proposed definition for bipolar spectrum disorder. Can J Psychiatry 2002;47:125-134.

29. Daruy-Filho L, Brietzke E, Lafer B, Grassi-Oliveira R. Childhood maltreatment and clinical outcomes of bipolar disorder. Acta Psychiatr Scand 2011;124:427-434.

30. Gilman SE, Ni MY, Dunn EC, Breslau J, McLaughlin KA, Smoller JW, et al. Contributions of the social environment to first-onset and recurrent mania. Mol Psychiatry 2015;20:329-336.

31. Etain B, Mathieu F, Henry C, Raust A, Roy I, Germain A, et al. Preferential association between childhood emotional abuse and bipolar disorder. J Trauma Stress 2010;23:376-383.

32. Young LT, Warsh JJ, Kish SJ, Shannak K, Hornykeiwicz O. Reduced brain 5-HT and elevated NE turnover and metabolites in bipolar affective disorder. Biol Psychiatry 1994;35:121-127.

33. Wiste AK, Arango V, Ellis SP, Mann JJ, Underwood MD. Norepinephrine and serotonin imbalance in the locus coeruleus in bipolar disorder. Bipolar Disord 2008;10:349-359.

34. Lee KS, Park YM, Lee SH. Serotonergic dysfunction in patients with bipolar disorder assessed by the loudness dependence of the auditory evoked potential. Psychiatry Investig 2012;9:298-306.

35. Ostermann J, Uhl I, Kohler E, Juckel G, Norra C. The loudness dependence of auditory evoked potentials and effects of psychopathology and psychopharmacotherapy in psychiatric inpatients. Hum Psychopharmacol 2012;27:595-604 